\title{
Por 13 Razones: \\ desafíos éticos frente al suicidio en una serie televisiva
}

\author{
13 reasons why | Brian Yorkey | Netflix | 2017 \\ Irene Cambra Badii* y Paula Mastandrea** \\ Universidad de Buenos Aires - CONICET
}

Recibido: 18 de junio 2017; aceptado: 1 de julio 2017

\begin{abstract}
Resumen
¿Existen verdaderamente "razones” para que una adolescente se suicide? Alejandro Ariel dijo en una oportunidad que "el suicidio es algo que no debería suceder", pero no en el sentido de una condena moral, sino para cuestionar su carácter de inevitabilidad. El tratamiento del problema a través de una serie televisiva permite un nuevo giro, en tanto las series resultan el relevo contemporáneo del cine para el gran público: diariamente, llegan a millones de espectadores en el mundo como antes lo hacían las novelas o la radio. Las historias que allí se presentan logran transmitir el pathos situacional y permiten un tratamiento inédito de los problemas. Pueden utilizarse en un marco psicoterapéutico (como en la cineterapia analítica) o bien como puntos de partida para el análisis ético-clínico de las ficciones en pantalla. La serie 13 Reasons Why (Netflix, 2017) ha sido un éxito justamente por plantear de una manera radicalmente diferente el fenómeno del "bullying”, introduciendo el horizonte de la muerte, planteando así interrogantes que alcanzan a la bioética y al psicoanálisis. En este trabajo se indagan algunas categorías cruciales, como la responsabilidad institucional y subjetiva, la violencia sexual, el acting out y el pasaje al acto. Se extraen así enseñanzas de la serie en tanto una forma cultural privilegiada que nos permite transmitir, interrogar y analizar de manera única y conmovedora las singularidades en situación.
\end{abstract}

Palabras clave: Cine | suicidio | bullying | psicoanálisis

13 Reasons why: Ethical challenges about suicide in a TV Series

Abstract

Can we really talk about "reasons" for a teen to commit suicide? Alejandro Ariel once said that "suicide is something that should not happen", not in the sense of a moral condemnation but to question its character of inevitability. The approach of teen suicide through a television series provides a new twist, given that series are the contemporary relay of cinema for the general public. Daily, television series reach millions of viewers in the world, as novels or radio used to do before. The stories presented manage to convey the situational pathos and allow an unprecedented treatment of the problems. They can be used in a psychotherapeutic framework (as cinematherapy, for example) or as starting points for ethical-clinical analysis of on-screen fictions. The series 13 Reasons Why (Netflix, 2017) has been a success precisely because it shows us the «bullying» phenomenon in a radically different way, introducing the horizon of death, thus raising questions for bioethics and psychoanalysis. In this paper we investigate some crucial categories, such as institutional and subjective responsibility, sexual violence, acting out and passage to act. The lessons of the series are therefore extracted as a privileged cultural form that allows us to transmit, interrogate and analyze in a unique and moving way the singularities in situation.

Keywords: Films | suicide | bullying | psychoanalysis

\section{El dispositivo estético de la serie televisiva: análisis desde una perspectiva $p s i$}

En la actualidad, por la potencia de su difusión, las series televisivas modelan la subjetividad de la época mucho más que otros fenómenos que gozan de mayor pres- tigio y consideración, como el cine (Gómez y Michel Fariña, 2012). De hecho, las plataformas online como Netflix y Amazon van, poco a poco, reemplazando la asistencia a las salas de cine. Allí no sólo se distribuyen filmes y series televisivas, sino que comienzan a crearse tales producciones, convirtiéndose en referentes inelu- 
dibles de la trama cultural de una época a través de sus plataformas online. Esto permite que las series se constituyan en un recurso muy valioso en el ámbito psi para analizar la subjetividad de la época ${ }^{1}$.

Durante el año 2017, la serie 13 Reasons Why (Brian Yorkey, 2017), producida por Netflix, ha tenido gran popularidad entre el público adolescente y adulto en general. El relato pone de manifiesto problemáticas actuales -como el fenómeno del bullying, la violencia escolar, el (des)trato de la diversidad sexual, el acoso y abuso hacia las mujeres- transitados por personajes adolescentes que generan en los espectadores una empatía e identificación respecto de esta etapa de la vida que no es atravesada sin conflictos.

La historia comienza a partir del suicidio de Hannah Baker, una adolescente de diecisiete años que ha decidido, previamente a quitarse la vida, grabar trece cassettes con su voz, donde queden registradas las razones de su muerte a los que hace alusión el título de la serie. Además de las grabaciones, Hannah ha dejado indicaciones específicas respecto de qué hacer con ellas: las cintas deben ser entregadas a cada una de las personas implicadas en esos relatos; de no ser así, las mismas serán divulgadas de manera pública. Así es como la serie va presentando, en cada uno de los trece episodios, a aquellos compañeros de colegio a quienes Hannah señala como responsables de su muerte.

El objetivo de este escrito no apunta a analizar los factores que han incidido en el éxito de 13 Reasons Why, sino más bien a indagar la propuesta narrativa de la serie en dos niveles de análisis: siguiendo lo que sucede con los personajes al interior del relato cinematográfico, y por otra parte investigando el planteo del director en sí mismo, a través del mensaje que se pretende transmitir y el dispositivo estético mediante el cual lo hace posible 2 . Toda la lectura está atravesada por una preocupación ética, tanto en lo que respecta a las responsabilidades de los actores institucionales como a las que emanan de cada uno de los personajes. Responsabilidades que por cierto alcanzan a los propios guionistas de la serie, como lo muestra claramente la decisión respecto de qué mostrar en la escena del suicidio, y cómo mostrarlo, lo cual ha provocado distintas reacciones entre los espectadores.

Comencemos con el esquema narrativo. Desde un primer momento, la serie genera interés por la estructura de su guión -que, a la manera de un rompecabezas, adelanta cuestiones de la trama como pequeños anuncios de suspenso. Si bien desde el comienzo sabemos que la protagonista terminará suicidándose, nos queda la pregunta: ¿por qué lo hizo? ¿Se pudo haber evitado?

Asimismo, la serie ubica la historia en el contexto de una secundaria estadounidense, con sus lockers, bailes de primavera, concursos para conocer a tu "compañero ideal para San Valentín”, equipos de baloncesto y porristas, división de los estudiantes entre populares y nerds, etc. La elección de la estética (tanto en relación con los planos cinematográficos como la iluminación) nos llevan a un mundo lila y rosa, con una banda de sonido que seduce a los melómanos con sus reversiones de clásicos con tono indie. Por una parte, esto genera un foco de atención claro en las historias de los adolescentes y en las intrigas de los pasillos de la escuela. Por otra parte, esto desplaza a los adultos fuera de la escena. Volveremos más adelante sobre esta cuestión.

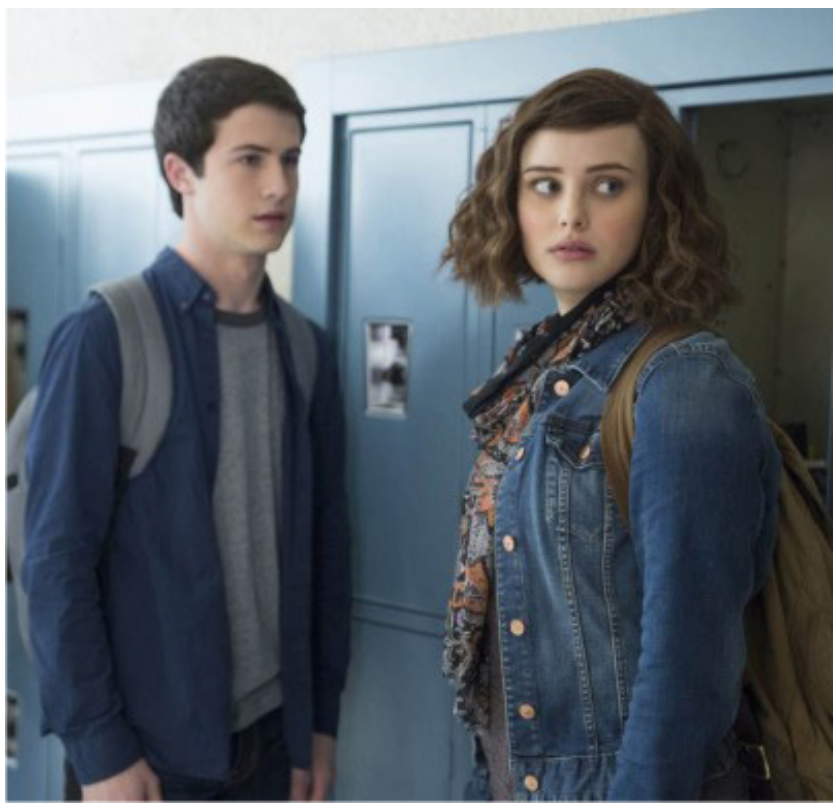

\section{El suicidio en la pantalla}

Demos ahora un paso más hacia una cuestión central dentro del análisis del dispositivo estético: la elección de mostrar explícitamente el suicidio de Hannah, lo cual fue argumentado por los mismos productores como un intento de representar el acto de la forma más explícita posible para desmotivar imitaciones.

La escena del suicidio, que acontece en el último episodio de la serie, resulta difícil de visualizar por cierta dimensión paradojal que provoca en el espectador el acercamiento a la problemática. Por un lado, hemos acompañado a la protagonista durante trece episodios, sabiendo desde el inicio que va a suicidarse, pero teniendo la secreta expectativa de que no lo haga. De 
alguna manera, sufrimos por ella, y con ella. Por otro lado, la escena pone de relieve que la angustia queda del lado del espectador y no de la protagonista, tanto en el primer plano de su rostro desangrándose en la bañadera, con las marcas de los cortes en sus muñecas, como en la escena en la que la encuentran sus padres al regresar a la casa.

¿Cuál es la incomodidad al ver esta escena? ¿Cuáles son los efectos que puede generar en los adolescentes que la miran?

La Organización Mundial de la Salud (2000) en su programa SUPRE (Suicide Prevention) recomienda estrictamente que la imagen de la víctima, el método empleado para tal fin, y la escena puntual del suicidio deben evitarse en las difusiones de noticias o ficciones sobre el suicidio. Esta indicación está motivada en el reconocimiento de que el suicidio está multideterminado, y que no sólo confluyen factores personales sino también familiares, sociales, educativos ${ }^{3}$. Si bien la serie puede entenderse como una invitación a repensar la problemática del suicidio (y del suicidio adolescente en particular), conjuntamente con la sugerencia a pensar en salidas a los problemas cotidianos que no sean tan drásticas ni violentas, todo el contenido estético de esta extensa escena radica en descripciones detalladas respecto del método utilizado, primeros planos de la protagonista en el momento del suicidio, y algunos elementos de idealización -leídos críticamente al interior de la serie, pero que no dejan de tener efectos. Estos elementos de idealización se dan en un tiempo posterior al del suicidio pero previo en el desarrollo de los episodios de la serie: así, vamos siguiendo el proceso de glorificación de Hannah como mártir, viendo cómo hacen casi un santuario del lugar donde guardaba sus libros, para llevarle flores y otras ofrendas recordatorias. Son estos elementos los que, conjuntamente con la visualización de una escena de suicidio sin sufrimiento subjetivo explícito, pueden ir en la vía de identificaciones riesgosas en personas que estén en un alto grado de vulnerabilidad ${ }^{4}$.

Asimismo, los compañeros de la escuela se ven a sí mismos como responsables de la muerte de Hannah sobre todo luego de escuchar los cassettes-, y esto puede resultar complejo por varias cuestiones: en primer lugar, porque expone y multiplica el efecto de culpa de los familiares y amigos de personas que han cometido un acto suicida (pensando cómo podrían haberlo evitado, por ejemplo); porque esconde la responsabilidad de los adultos en tal situación, y porque vela el mensaje de la serie advirtiendo que este suicidio es una "lección" para que los demás “aprendan”. He aquí una peligrosa advertencia para los demás: considerar el suicidio como una lección deja un resto subjetivo que será imposible de retomar en el contenido de la serie.

\section{El deslizamiento entre bullying y suicidio}

Si bien la pregunta central de la serie gira en torno al porqué del suicidio de Hannah -como si fuera posible comprender las razones últimas de un suicidio-, todo el relato de la serie pone de manifiesto la trama de sufrimiento escolar y abusos que tantas veces quedan invisibilizados institucionalmente. Explícitamente, en la serie se menciona el acoso escolar como uno de los posibles motivos del suicidio de Hannah, razón por la cual se produce un juicio contra la escuela, responsable de tal situación. Los jóvenes destinatarios de las cintas de cassettes de Hannah van a declarar y -por primera vez- se visibiliza la trama de abusos frente a los adultos.

Ahora bien, ¿se trata efectivamente de un fenómeno de bullying? ¿Es este fenómeno presentado por la serie como la causa última del suicidio de su protagonista? ¿Resulta correcto establecer esa relación unicausal entre una y otra cuestión?

En la actualidad, en término bullying ha adquirido gran popularidad en el dialecto cotidiano para referirse a cualquier situación de violencia ejercida en la escuela. Sin embargo, es necesario realizar una diferenciación conceptual respecto de los conflictos que pueden ocurrir entre los jóvenes en este ámbito institucional.

El fenómeno del bullying fue definido por primera vez por Dan Olweus (1998) como una conducta de persecución física o psicológica que se realiza en reiteradas oportunidades “entre iguales", es decir, desde un alumno hacia otro. Las características específicas que distinguen al bullying de otros tipos de violencia son: que implica un comportamiento agresivo con el fin de hacer daño; que el acoso debe existir de manera reiterada y aún fuera de la escuela; que debe suponer una relación interpersonal en la que existe una asimetría de poder. Olweus afirma que dichas situaciones de abuso ocurren bajo la complicidad o indiferencia de los otros compañeros. Además, la gravedad de los hechos en la actualidad podría deberse a la falta de una terceridad, que debería provenir de los adultos a cargo (Travnik, 2014).

En el caso de 13 Reasons Why, encontramos que existen múltiples y variados actos de violencia contra Hannah: violencia verbal; violencia sexual; violencia 
psicológica y violencia relacional y exclusión social. Cada una de estas situaciones se traduce en la adolescente como inseguridades, baja autoestima, tristeza y padecimiento, y un consecuente alejamiento de los pocos vínculos sociales que había logrado establecer cuando llega a su nueva escuela.

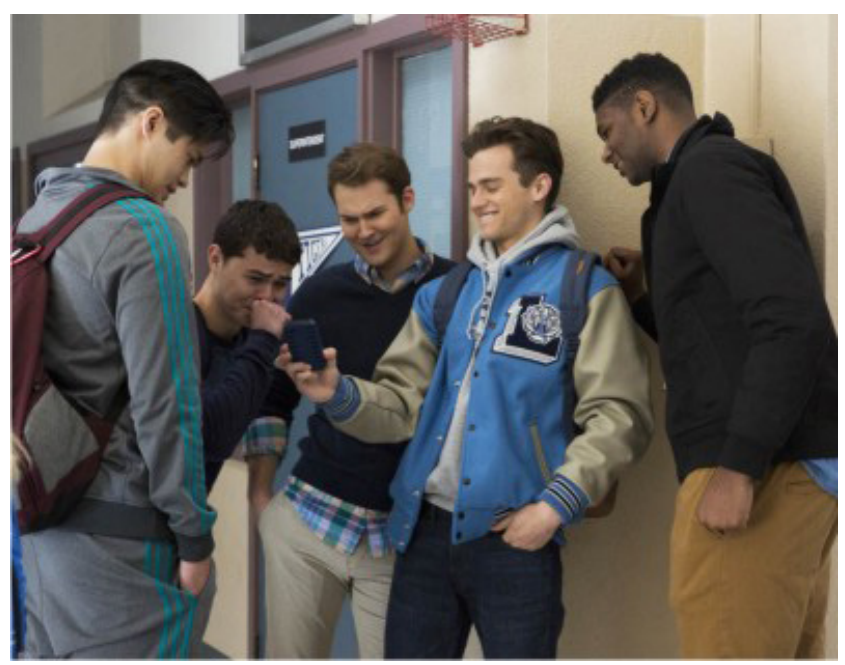

En la actualidad, por la popularidad del término y su impacto social, tiende a considerarse toda conducta violenta como bullying, sin embargo, es necesario poder establecer una distinción conceptual respecto de las distintas situaciones de padecimiento que acontecen en el dispositivo escolar. Poder nombrar los hechos de manera correcta evita el reduccionismo simplista que tiende a destinar el mismo tratamiento a todas las situaciones, $y$ permite pensar estrategias de prevención y cuidado frente a las conductas adolescentes de riesgo. ¿Hannah Baker sufre bullying? Por supuesto que sí, pero no todo lo que le ocurre puede ubicarse en la misma categoría. Dentro de las situaciones que la adolescente cuenta, encontramos aquellas en las cuales efectivamente existe un abuso sostenido en el tiempo, con intención de hacer daño y con asimetría de poder: por ejemplo, cuando uno de los jóvenes populares de la escuela, y sus amigos (carilindos y atletas), divulgan una foto de Hannah que se presta a confusión respecto de un supuesto acto sexual cometido con el primero. A raíz de ello, el acoso se sostuvo en el tiempo a través de distintas conductas y frases de los varones de ese grupo para con ella. Asimismo, podríamos suponer cierta asimetría de poder al considerar que dicho grupo de amigos representaban a "los populares" de la escuela, lo cual les daba gran influencia sobre el resto del alumnado, que se convencía de su versión sobre Hannah sin acercarse a ella para conocer su experiencia sobre tal situación. Sin embargo, encontramos otras si- tuaciones en los cassettes que también tuvieron un fuerte impacto en la protagonista y le produjeron padecimiento, situaciones violentas pero que no serían una expresión del bullying. Entre ellas podemos ubicar la violencia verbal que comete su amiga Jessica contra ella, cuando la increpa suponiendo que está coqueteando con su novio, o cuando Courtney la excluye y aísla de su grupo porque teme que se ponga en evidencia su homosexualidad oculta frente a toda la escuela.

Si no se lleva a cabo un análisis que pueda diferenciar las distintas situaciones que llevan a Hannah hacia el suicidio, la problemática del bullying queda cercada, simplificando sus términos: se enfoca sobre un responsable en particular, y se asocia unicausalmente el bullying y el suicidio. El estudiante que abusa sexualmente de Hannah y otra compañera, o el que la humilla difundiendo su fotografía, pueden ser vistos entonces como chivos expiatorios, únicos referentes de la problemática cuando en realidad son la punta de un iceberg que, además, no se conecta unicausalmente con la decisión del suicidio. El problema de la serie parece agotarse entonces en una lectura lineal que termina velando la compleja trama del padecimiento psicosocial ${ }^{5}$.

\section{La angustia en la adolescencia ${ }^{6}$}

Por otra parte, la trama de la adolescencia también puede analizarse a través de la serie, ya que funciona a la manera de un sub-texto: está presente, pero no es analizada en su especificidad. De algún modo, pareciera ser que la historia de Hannah Baker podría ser la de cualquier persona (la indagación de las razones de un suicidio nos llevaría por esa vía). Sin embargo, la adolescencia como etapa vital tiene especificidades que vale la pena tener en cuenta para el análisis de la serie.

Como ya es sabido, la adolescencia es un momento de transformaciones y cambios. En esta etapa se enfrenta el cambio del cuerpo infantil ya comenzado en la pubertad, la búsqueda de una identidad propia y diferenciada de los padres, se fortalecen los vínculos con los iguales, al mismo tiempo que se necesita de su aceptación y reconocimiento. Grassi (2010) denomina lo puberal-adolescente a los trabajos psíquicos que implican un potencial saludable de cambio en esta etapa, promoviendo un des-orden del statu quopromotor de neoorganizaciones. Lo puberal adolescente trabaja para la incorporación y homogenización de lo nuevo, lo distinto (Maroño, 2016), que proviene de distintas fuentes: un campo in- 
tra-subjetivo (cambios corporales y vicisitudes de la historia personal), un campo intersubjetivo (relaciones familiares, conjunto de los coetáneos y círculo más amplio con quienes se comparte un período histórico-político y social) y un campo que toma una dimensión trans-subjetiva ya que conecta con las generaciones precedentes vía transmisión generacional de la vida psíquica.

En esta línea, vemos que Hannah Baker manifiesta estas problemáticas a lo largo de los trece episodios de la serie, al igual que sus compañeros. Sin embargo, en su caso la tristeza va calando hondo y se suceden situaciones de mayor gravedad -desde la primera soledad por no conocer a nadie en la escuela nueva, pasando por el ir perdiendo o alejándose de los amigos que va coincidiendo, hasta las escenas de abuso sexual en donde la protagonista queda claramente afectada, y la angustia sin tramitar la va ahogando cada vez más.

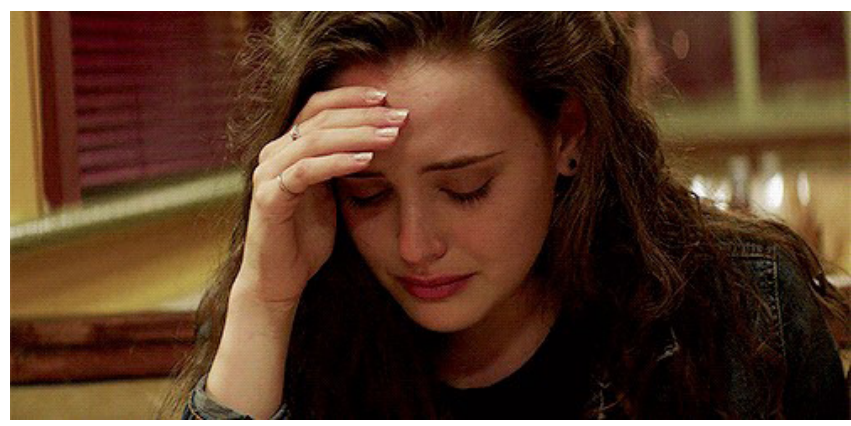

Para analizar esta cuestión, encontramos pertinentes los desarrollos de Lacan (1963) respecto de las patologías del acto, acting out y pasaje al acto, como modos de respuesta frente a la angustia. Sin embargo, es necesario aclarar que la serie no tiene rigor clínicoen sí mismo, y que por lo tanto las puntualizaciones que aquí se hacen pueden resultar de alguna manera provisorias, en tanto y en cuanto no contamos con mayores detalles del devenir subjetivo de la protagonista más allá de lo que la serie se empeña en demostrar.

Como sabemos, el acting out implica el armado de una escena fantasmática que siempre se dirige a un Otro, se trata de una mostración que no entra en el terreno del decir. Por el contrario, el pasaje al acto supone una borradura del sujeto de esa escena, es decir, se deja caer del marco de su realidad fantasmática identificándose como resto.

En los adolescentes de la serie, encontramos diversas situaciones que podrían interpretarse como acting out, llamados al Otro: abuso de sustancias; enfrentamientos violentos entre ellos, conductas de riesgo, entre otras. Las mismas podrían leerse como un llamado al Otro de la ley, es decir, como una demostración del sentimiento de abandono que genera la falta de límites y de acompañamiento por parte de los adultos que deberían custodiar el proceso de cambio que se encuentran atravesando.

Hannah llama al Otro en las experiencias que se relatan en los cassettes. Sin embargo, al momento de grabar las cintas ya no hay un Otro que la sostenga en ese llamado, sino que más bien se vuelven una maldición, una manifestación de su enojo, una culpabilización hacia quienes no pudieron escucharla, una mortificación para quienes no le han respondido?.

Cuando no logra que nadie la aloje, el acting deviene pasaje al acto: ya no hay a quién llamar, y el sujeto cae de la escena. La escena del suicidio es de una profunda desdicha, donde Hannah muere en silencio y en soledad, pero la angustia queda del lado del espectador.

\section{La escucha de los adultos}

Resulta llamativo que en el relato de la serie los adultos estén desplazados del foco de atención, desresponsabilizados en la trama que lleva al suicidio de Hannah, como si las historias vividas por los adolescentes nos confrontaran con sus dramas existenciales sin contextualizarlos en una determinada etapa vital que requiere de la guía y la atención de los mayores.

Duek (2016) plantea que el período adolescente implica una reorientación subjetiva que acontece en el mismo seno del yo, en la base identificatoria del ser mismo. En este proceso, es necesario que los modelos previos caigan ya que, a través del repudio a los baluartes familiares identificatorios, el adolescente busca diferenciarse. $\mathrm{Al}$ mismo tiempo, esta actitud es un modo de sostenerse frente al dolor y sufrimiento inconsciente que implica el duelo por el "ser infantil", por los padres de la infancia. El fin de la infancia requiere asesinar simbólicamente a esos padres para confrontar, destituir y dar espacio a una nueva organización psíquica.

Sin embargo, para que este proceso se lleve a cabo de manera saludable, el adolescente requiere del reconocimiento de los otros que medien el orden simbólico, que lo nombren y lo anoten en el paisaje del entretejido de los semejantes.

El análisis del período de lo puberal-adolescente no debe desconocer que las condiciones socioculturales en las que vivimos funcionan a modo de red de prácticas que intervienen en la constitución misma de los tipos subjetivos reconocibles en una situación sociocultural 
específica (Lewkowicz, 2004). En este sentido, la posmodernidad se caracteriza por la ausencia de garantes simbólicos, aquellos que en la modernidad funcionaban a modo de Gran Sujeto, como instancias de terceridad ante quien el sujeto pueda verdaderamente presentar una demanda, formular una pregunta o hacer una objeción. El sujeto de la posmodernidad debe definirse a sí mismo, lo cual lo confronta con la angustia de la autofundación, ya que un sujeto definido autorreferencialmente es también un sujeto penetrado por la ausencia de definición.

Las condiciones sociales que se le ofrecen a los trabajos puberales-adolescentes, ahí donde la subjetividad debería advenir, dan cuenta de una dimensión de futuro que se des-dibuja cuando las prácticas de consumo y el discurso mediático los atraviesan silenciosamente, penetrando de un modo persistente y tenaz en la cotidianeidad. Nuevos rasgos se producen en la subjetividad actual, rasgos desligados a las funciones tradicionales de la familia o la escuela. La velocidad y la aceleración son modos de estar en esta era de la fluidez (Lewkowicz, 2004), produciendo subjetividades fragmentadas y vacías de significación (Angelli, Altobelli y Otero, 2009).

Es a partir de estas condiciones de época que la relación entre los adolescentes y los adultos se ha visto modificada. Tal como observamos en la serie, ninguno de los adolescentes confía sus problemas a sus padres, es decir, éstos no aparecen como posibles vías de solución frente a las problemáticas y conflictos que los jóvenes atraviesan.

En base a los desarrollos de Duek (2016), consideramos que dicho modo de vinculación corresponde a un rasgo típico de la etapa evolutiva en la que se encuentran los protagonistas, al mismo tiempo que responde a la pérdida de referentes simbólicos propios de la posmodernidad.

Sin embargo, lo que resulta interesante para analizar a partir de la historia presentada, es el hecho de que los adultos parecen no tener recursos para alojar y acompañar a los jóvenes en esta lenta y progresiva desvinculación de las ligazones emocionales y familiares. El resultado es un grupo de adolescentes "sueltos", a merced de sus impulsos y haciéndose cargo por sí mismos de situaciones complejas.

\section{La responsabilidad del consejero escolar}

El único adulto a quien Hannah recurre es el consejero escolar, el señor Porter, quien se convierte así en el destinatario del último cassette. El episodio final de la se- rie está enfocado en la decisión que toma Hannah de finalmente quitarse la vida, luego de haberse entrevistado con el consejero escolar. El montaje de la secuencia, que alterna los diálogos de Hannah y de Clay con el consejero, acentúa nuestra identificación con los protagonistas adolescentes.

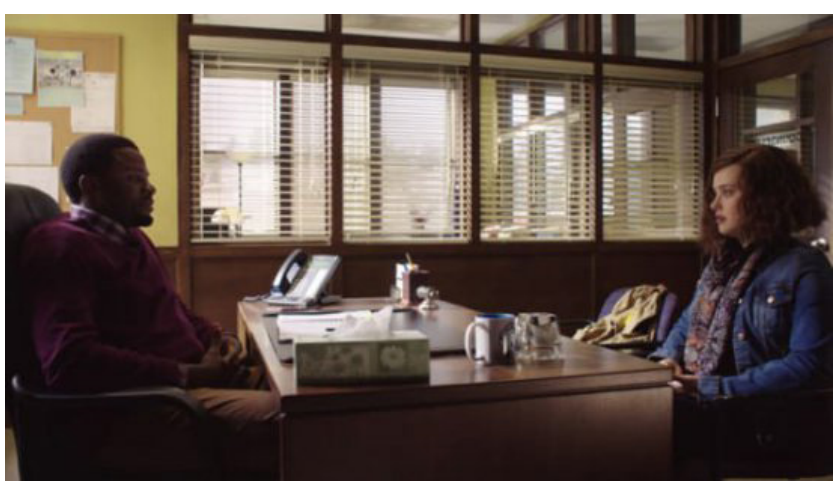

¿Qué espera encontrar Hannah en esa entrevista, y qué le ofrece Porter? En palabras de la adolescente, es la última oportunidad que le da a la vida, al ir a pedir ayuda por primera vez a un referente adulto, no a un par; y relatar el abuso sexual sucedido unos días atrás, para el cual no encuentra consuelo. Espera que el consejero la detenga cuando desliza sus intenciones de matarse, y también cuando se va del consultorio, pero éste parece limitarse a un accionar que toma en cuenta la normativa sin ninguna apreciación de la dimensión clínica (Salomone, 2006).

La escena es rica en anotaciones. En primer lugar, porque nos permite indagar la cuestión de la responsabilidad del consejero escolar -figura que no existe en Argentina, pero que evoca la de un psicólogo ${ }^{8}$. ¿Cuáles son los límites y las responsabilidades de esta función en la escuela? ¿Cuáles son las competencias que debe tener el profesional que ocupe este rol?

Por otra parte, podemos situar una mala praxis en esta actuación profesional ${ }^{9}$. La adolescente nota que el consejero no la escucha. El teléfono de Porter no deja de sonar-evidencia de que tiene problemas familiares que ocupan su cabeza en lugar de estar in situ. Ella misma lo habilita para que atienda la llamada, cuando la acción misma de ir a ver a Porter es una llamada de atención sobre lo que le está sucediendo.

En el devenir de la serie y de la historia de Hannah, vemos que el consejero escolar no es simplemente " $u n$ adulto más" que falla y no la escucha. Es el único a quien Hannah acude en busca de ayuda. El hecho de no poder alojar al Sujeto en esa situación, cuando fue requerido en tanto profesional, duplica el daño. 
Nasio (2011) plantea que existe una cierta disposición mental con la que el profesional debe abordar a un adolescente en crisis: a) tiene que tener ganas de comunicarse con él y tratar de sentir lo que él siente conscientemente, en primer lugar y luego, si se tiene la formación adecua$\mathrm{da}$, ir al plano inconsciente; $\mathrm{b}$ ) sentirse uno mismo disponible y que nos sienta disponibles para recibirlo sin reservas, tal como es. No juzgarlo; c) no jugar a ser amigos ni ser demagogo. El joven espera encontrar un adulto que, por su diferencia y por su presencia, le recuerde los límites de la realidad y al hacerlo, logre calmarlo; d) Mostrarle que se toma en serio lo que dice e) alentarlo a hacer preguntar sobre cualquier tema; y f) no se debe tomar ninguna iniciativa sin pedirle su opinión.

Vemos en la escena que nada de esto ocurre. Si bien es claro que acude al consejero para pedirle ayuda, Hannah comienza dando rodeos a la situación, sin poder avanzar en el diálogo. Señala que nada parece importarle, que no es quien sus padres necesitan que sea, que no tiene amigos. Cuando la charla prosigue, la vemos angustiarse en dos ocasiones puntuales: cuando el señor Porter le señala que "parece que hay algo que necesitas y no lo estás obteniendo" (y ella responde con lágrimas en los ojos: "Necesito que todo se detenga") y cuando Hannah le dice que se avergüenza de lo que pasó en la fiesta, una semana atrás.

Tenemos aquí la clave para el consejero escolar: ¿qué es lo que la angustia de esa situación? ¿Cómo podemos actuar allí? Sin tener en cuenta esta angustia que comienza a desbordarla (y frente a la cual caerá), el señor Porter se centra en primer lugar en las acciones de Hannah frente al abuso: “ ¿le dijiste que se detuviera?, ¿le dijiste que no? (...) Quizás lo consentiste y luego cambiaste de opinión" para luego centrarse en aspectos ligados a la denuncia legal: "¿Deberíamos involucrar a tus padres o a la policía?”, “¿Cuál es el nombre del chico?”. Todas sus intervenciones radican en los aspectos normativos del caso, lo que debería hacerse siguiendo el protocolo estandarizado de las situaciones de abuso en la institución escolar. Así, repite a Clay y a Hannah: "cuando un estudiante es abusado, mi función requiere que vaya a la Policía, pero necesito saber exactamente qué pasó y quién lo hizo".

Al ubicar estos procedimientos normativos por encima de los efectos subjetivos en Hannah, aplasta toda posibilidad de elaboración incluso explícitamente: "Si no puedes darme un nombre, si no quieres presentar cargos contra él, si no estás segura de que puedas hacerlo, entonces solo queda una opción. No quiero ser insensible,
Hannah... pero puedes seguir adelante". Es interesante que en inglés, Hannah le replica diciendo que se trata entonces de "Do nothing", que significa, literalmente, "hacer nada”, puesto que se trata de una oración afirmativa. En efecto, no se trata de negar una acción posible, sino de que Porter, justamente, propone "hacer nada".

Es Clay quien interpela a Porter en la conversación que mantiene luego de escuchar la última cinta de Hannah. Señala como generalidad ("todos debemos responsabilizarnos, todos podemos hacerlo mejor") lo que en realidad resulta un mensaje dirigido especialmente al consejero: es él quien debería haberlo "hecho mejor".

Mencionamos entonces una breve reflexión sobre la responsabilidad profesional. Por supuesto que se pueden pasar por alto las "señales". No todos los profesionales pueden advertir el devenir subjetivo de todos sus pacientes. Se cometen errores. Pero allí no radica la responsabilidad del consejero. Como espectadores, no debemos distraernos en esta cuestión ya que desde el inicio de la serie conocemos el desenlace del suicidio. Sabiendo de este final, quizás resulta más fácil leer los gestos de Hannah. Sin embargo, hay un gesto clave en Clay: creerle a Hannah en los trece cassettes que acaba de escuchar, confiar en su palabra, decir en voz alta el nombre de quien la violó, suplementar la escena con un nuevo cassette y enfrentar así a Porter con una acción pendiente.

\section{A modo de cierre}

Más allá de las controversias que ha despertado la serie -con sus admiradores y detractores- el escenario de esta narrativa cinematográfica nos ofrece una nueva ocasión para repensar las coordenadas de la adolescencia, el bullying y la violencia escolar, el abuso sexual y el suicidio, y la responsabilidad profesional del psicólogo en estas situaciones, alejándonos del impacto inmediatista y de concepciones moralistas que cierren la vía de problematización de la cuestión.

El testimonio de Hannah Baker, a partir de la materialidad de los cassettes (imposibles de borrar, e indelegables para sus destinatarios), posibilita empezar a pensar la responsabilidad frente al semejante, el sufrimiento ajeno, y las experiencias límite en la adolescencia. Asimismo, si bien la serie se llama Por 13 Razones, entendemos que los motivos que llevan a semejante conducta resisten cualquier enumeración. Las motivaciones inconscientes -en la línea de lo desarrollado en función del acting out, el pasaje al acto y la adolescencia- pueden ofrecernos 
distintas vías de análisis para abordar la cuestión en una complejidad que excede a la propia serie.

Esta propuesta va por lo tanto más allá, sin perseguir un objetivo meramente educativo o de prevención del riesgo suicida, e incluso sin detenernos únicamente en la reprobación de la escena explícita del suicidio de la protagonista. Se podría pensar que el deslizamiento más espinoso que propone la serie no radica en la indiferencia- ción entre bullying y violencia escolar, o entre bullying y suicidio, sino justamente al introducir la posibilidad cuantitativa de que existan efectivamente una (o dos, o trece) razones para suicidarse.

Nuestro desafío radica, entonces, en poder ir más allá del dispositivo montado por los realizadores, sosteniendo una mirada crítica que permita construir preguntas allí donde la serie arriesga agotarlas en apenas trece respuestas.

\section{Referencias}

Assef, J. (2013). La Subjetividad Hipermoderna. Una lectura de la época desde el cine, la semiótica y el psicoanálisis. Buenos Aires: Grama Ediciones

Duek, D. (2016). Intersecciones: Los otros, los jóvenes y los discursos sociales. Actualidad Psicológica. Enero 2016.

Grassi, A. (2010) Adolescencia: reorganización y nuevos modelos de subjetividad. En:Entre niños, adolescentes y funciones parentales. Psicoanálisis e interdisciplina (pp. 29-44). Buenos Aires: Editorial Entreideas.

Gómez, M.; Michel Fariña, J.J. (2012). Series: una interpretación del síntoma. Ética y Cine Journal, 2(2), 9-10.

Lacan, J. (1963). El Seminario 10. La Angustia, Clase IX, Buenos Aires: Paidós, 2006.

Maroño, M. R. (2016). Cualidades fácticas y psíquicas del proceso puberal en niñas. Tesis de Doctorado en Psicología. Universidad del Salvador. Inédita.

Michel Fariña, J., Salomone, G. et al (2007): Logos, Pathos, Ethos: Developing Ethical Sensitivity in Argentinean Teachers using the Racial and Ethical Sensitivity Test (REST). Annual Meeting of the Association for Moral Education, New York University, November 2007.

Nasio, J.D. (2011). Cómo actuar con un adolescente difícil. Buenos Aires: Paidós.

Olweus, D. (1998). Conductas de acoso y amenaza entre escolares. Madrid: Ediciones Morat.

Smith, P.K, Mahdavi, J., Carvalho, C., Fisher, S., Russell, S., y Tippett, N. (2008). Cyberbullying: Its nature and impact in secondary school pupils. Journal of Child Psychology and Psychiatry, 49, 376-385.

Travnik, C. (2014) El acoso escolar: una encerrona trágica. Ética y Cine Journal, 4(2), 53-57

World Health Organization. Prevención del suicidio: Un instrumento para los medios de comunicación [Internet]. Geneva: World Health Organization; 2000. Disponible en: http://www.who.int/mental_health/media/media_spanish.pdf

World Health Organization (2012). Prevención del suicidio: un imperativo global. Disponible en: http://www.who.int/mental_health/suicide-prevention/es/

World Health Organization (2014). Preventing suicide: A global imperative. Disponible en: http://apps.who.int/iris/bitstre am/10665/131056/1/9789241564779_eng.pdf

\footnotetext{
1 Recordemos que, desde el psicoanálisis, es el goce y el discurso lo que constituye la subjetividad de una época. Comprender el discurso de una época, conocer los significantes-amo que rigen el lenguaje de un tiempo, es establecer las nociones de un decir hegemónico que organiza ciertas prácticas de los sujetos que comparten un tiempo y un espacio, es decir, comprender una modalidad de gozar predominante propuesta por el discurso (Assef, 2013).

2 Además, podríamos agregar: incluso con lo que va más allá de su intencionalidad consciente como realizador cinematográfico.

3 Como sabemos, el suicidio es una problemática mundial de salud pública, y ha aumentado progresivamente sus tasas en los últimos 50 años. Actualmente mueren alrededor de un millón de personas al año y se considera que en el 2020 esta cifra llegará a 1,5 millones (World Health Organization, 2014). Asimismo, el suicidio adolescente requiere una especial atención: es la segunda causa de muerte en personas con edades entre los 15 y los 29 años (World Health Organization, 2012).
}

$4 \quad$ Al momento de publicación de este artículo, se conocen dos casos de adolescentes identificados con la protagonista de la serie: un joven peruano de 23 años que dejó cassettes grabados en los momentos previos al suicidio, y una adolescente dominicana de 11 años que se cortó los brazos y manifestó su intención de suicidarse. Fuentes: http://www.playgroundmag.net/noticias/actualidad/ peruano-suicida-imitando-Rasons-Why_0_1987601233.html y http://www.listindiario.com/la-republica/2017/04/30/463955/nina-intento-suicidarse-influenciada-por-serie-tv

5 Asimismo, es interesante notar que el motor narrativo de la serie es el personaje de Clay Jensen, un adolescente enamorado de Hannah, quien va escuchando los cassettes y acompaña el movimiento del espectador al caer en cuenta de lo que le sucedía a nuestra 
protagonista. Clay se muestra culpable desde un primer momento, aunque como sabremos luego, Hannah no lo ubica como responsable de su muerte. Como señala Michel Fariña, la culpa vela y devela: en este caso, la responsabilidad pendiente de Clay no se encuentra en relación con la trágica decisión de Hannah sino más bien con su propia cobardía subjetiva al retroceder ante su deseo y dejarla ir.

6 Agradecemos los aportes de Eduardo Laso y Juan Jorge Michel Fariña para pensar estos apartados.

7 De alguna manera, esta maldición recae sobre el espectador: así como sus compañeros “deberían haber visto las señales”, en el mensaje explícitamente educativo de la serie nosotros también nos convertimos en deudores de quienes sufren, de quienes no son escuchados, de quienes son silenciados. Sin embargo, esta lectura esconde la hiancia que se produce entre la decisión de matarse y el "echarle la culpa" a los demás, esperando que resulten afectados por ese llamado de atención. De alguna manera, una vez más, la serie sigue la lógica de las fantasías adolescentes de los personajes y la replica en el argumento mismo.

8 Respecto de las situaciones dilemáticas del rol del consejero escolar, cfr. el trabajo de Michel Fariña et al. (2007) sobre sensibilización ética en el ámbito educativo y el uso del REST (Racial and Ethical Sensitivity Test) para estos escenarios. Se trata de personas clave en la dinámica escolar, ya que los estudiantes acuden a ellos cuando tienen situaciones difíciles de manejar en sus casas, con sus pares o con sus docentes. El consejero se convierte así en un articulador institucional que debe trabajar con situaciones dilemáticas, tanto a nivel personal como escolar.

9 Sostenemos la diferencia entre mala praxis y falla ética fundamentando que en la primera no hay una intencionalidad por parte del profesional, aun cuando los resultados de los errores sean devastadores (Michel Fariña, 1992). 